\title{
QUESTÕES ATUAIS ACERCA DO TRANSTORNO DE DÉFICIT DE ATENÇÃO/ HIPERATIVIDADE
}

\section{Current Matters About Attention Deficit Hyperactivity Disorder}

\author{
André Luiz Monézi Andrade ${ }^{1}$ \\ Alfredo Lohr Júnior ${ }^{2}$
}

\section{Resumo}

O transtorno de déficit de atenção/hiperatividade (TDAH) é um transtorno mental freqüentemente encontrado entre as patologias neuropsiquiátricas mais comuns na atualidade. Sua prevalência varia entre 3 a 5\% das crianças em idade escolar, e aproximadamente $4 \%$ da população adulta. Sua etiologia ainda não está totalmente esclarecida, mas vários estudos sustentam que o TDAH parece ter causas multifatoriais, que abrangem desde aspectos bioquímicos e neurológicos, até psicológicos e socioambientais. O diagnóstico do TDAH fundamenta-se em critérios clínico-comportamentais, dependendo da história clínica do paciente, informe dos pais e da escola e uso de questionários formais. O DSM-IV reconhece três subtipos de TDAH, o subtipo desatento, subtipo hiperativo/impulsivo e o subtipo combinado. Mais de $70 \%$ de pacientes com este transtorno que procuram ambulatórios especializados apresentam alguma comorbidade, dentre elas, destacam-se o distúrbio de conduta, comportamento desafiador opositivo, dislexia, distúrbios da linguagem e ansiedade. Os tratamentos para o TDAH podem ser por meio de diversos tipos de fármacos, dentre eles destacam-se as anfetaminas. Abordagens psicossociais, psicoeducacionais, cognitivo-comportamental e tratamentos combinados (terapia medicamentosa e não medicamentosa) são outras modalidades de tratamento. Os resultados apontam uma eficácia na redução dos sintomas do transtorno por ambas formas de intervenção. O tratamento combinado é o mais recomendado para o portador do transtorno por conseguir contemplar uma maior quantidade de variáveis possivelmente envolvidas com a aquisição e/ou manutenção dos sintomas relacionados ao TDAH. Este trabalho retrata de forma sucinta e objetiva questões referentes ao conceito, diagnóstico, etiologia, tratamentos farmacológicos, não farmacológicos e combinados para o TDAH.

Palavras-chave: Transtorno de Déficit de Atenção/Hiperatividade; Desatenção; Hiperatividade.

1 Acadêmico do Curso de Psicologia do Centro Universitário Positivo. Centro Universitário Positivo, Centro Psicológico - Núcleo de Neurociências. Rua Professor Parigot de Souza, 5300. Campo Comprido, Curitiba-PR CEP: 81280-330. E-mail: andreandrade@unicenp.edu.br

2 Neuropediatra, Mestre em Medicina Interna - UFPR. Professor do Centro de Ciências Biológicas e da Saúda da PUCPR e do Centro Universitário Positivo. Chefe da Unidade de Neuropediatria do Hospital Pequeno Príncipe. E-mail:

a.lohrjunior@unicenp.edu.br 


\section{Abstract}

Attention Deficit Hyperactivity Disorder (ADHD) is a mental disorder found between the most common neuropsychiatry pathologies nowadays. Its occurrence varies between $3 \%$ and $5 \%$ of the children in scholar years, and approximately $4 \%$ of the adult population. ADHD etiology is not yet completely clarified, but a lot of researches support that this disease seems to have multifactor causes, that range since biochemical and neurological aspects to psychological and socio-environmental ones. ADHD diagnosis bases itself in clinicalbehavioral criterions, depending on the patient's clinical history, parental and school informing and formal questionnaire usage. The DSM-IV recognizes three ADHD subtypes, the absent-minded subtype, hyperactive/ impulsive subtype and combined subtype. More than $70 \%$ of the ADHD patients that look for specialized ambulatories present some comorbidity, among them, outstands behavior disturb, oppositional defiant disorder, dislexy, language disturb and anxiety. Among the different types of medicines for the ADHD treatment are distinguished the amphetamines. Other approaches of treatments can be as following: psychosocial, psychoeducational, cognitive-behavioral therapy and combined treatments (medical and non medical therapy). The results indicate a effectiveness in the reduction of the symptoms of the disorder for both forms of intervention. The combined treatment is the most effective one for ADHD since it contemplate the larger number of variables associated with the acquisition and/or maintenance of the symptoms related with the ADHD. This paper shows briefly and objectively the issues related with the concepts, diagnosis, etiology, pharmacologic and non pharmacologic treatments and also combined treatments for ADHD.

Keywords: Attention- deficit hyperactivity disorder; Inattention; Hyperactivity.

\section{Introdução}

O Transtorno de Déficit de Atenção/Hiperatividade (TDAH) é um transtorno mental caracterizado por sintomas de desatenção, hiperatividade e impulsividade, sendo um dos transtornos mais comuns na infância e adolescência (Daley, 2006; Voeller, 2004). Sua prevalência estimada varia entre 3 a $5 \%$ das crianças em idade escolar (Fabiano \& Pelham, 2003) e aproximadamente 4\% da população adulta (Biederman, Milberger, Faraone, Guite, \& Warburton, 1994).

A primeira descrição médica do TDAH foi feita no início do século XX pelo médico inglês George Still, que o definiu como um efeito no controle da moral. Still defendeu a hipótese de que esta condição teria como base um substrato biológico que poderia ser hereditário e/ou relacionado a uma lesão cerebral adquirida e não como conseqüência de uma má educação ou depravação como até então se acreditava (Monteiro, 2005). A partir dessa descrição, o TDAH recebeu diversas denominações ao longo dos anos. Na década de 40, com base nas percepções de Still, cogitou-se a hipótese que TDAH seria decorrente de uma "lesão cerebral mínima", designação esta que pendurou até o início da década de 60, quando novos estudos sugeriram uma relação do TDAH mais próxima a disfunções neurofisiológicas do que estruturais, modificando sua nomenclatura para "disfunção cerebral mínima” (Rohde \& Halpern, 2004).
Atualmente, os sistemas classificatórios mais modernos em Psiquiatria propõem a terminologia Transtorno de Déficit de Atenção/Hiperatividade (DSM-IV, 2002) e Transtornos Hipercinéticos (CID10, 1993).

\section{Etiologia}

As investigações científicas mais recentes para a determinação da etiologia do TDAH atingem um amplo campo que abrange desde aspectos bioquímicos e neurológicos até psicológicos e socioambientais.

Várias evidências sugerem que o TDAH é caracterizado por uma disfunção na transmissão dopaminérgica nos lobos frontais e nos gânglios basais, especificamente no estriado (Vaidya et al., 2005). Estudos realizados por meio de modernas técnicas de neuroimagem demonstraram que o cérebro de crianças com TDAH possui uma redução tanto do metabolismo ( $\backslash$ o "Click to search for citations by this author." Amen \& $\backslash \mathrm{O}$ "Click to search for citations by this author." Carmichael, 1997) quanto do volume (Castellanos et al., 2002; Mataro, Garcia-Sanchez, Junque, Estevez-Gonzalez, \& Pujol, 1997) das regiões estriatal, cerebelar e frontal. Nesta última, sabe-se que uma área localizada no lobo frontal (região orbital frontal) possui uma função ímpar em vários aspectos sintomatológicos do TDAH (Aman, Roberts \& Pennington, 1998). 
Tal fato ocorre porque esta região não consegue inibir com eficácia todos os estímulos recebidos pelo cérebro, conseqüentemente o indivíduo não consegue se manter concentrado em um estímulo específico por muito tempo (déficit de atenção). Embora exista uma forte correlação entre certas áreas cerebrais, cada qual relacionada a um conjunto específico de sintomas, ainda não existem estudos demonstrando de forma precisa todos os processos neurobiológicos envolvidos no TDAH (Excoffier, 2006).

Alterações no funcionamento de neurotransmissores, tais como a dopamina, noradrenalina e serotonina têm sido fortemente relacionadas ao transtorno (Pliszka, 2005). O sistema dopaminérgico, particularmente a via mesolimbocortical - nigroestriatal, está relacionado com a atividade motora, funções cognitivas como fluência verbal, aprendizado, sustentação e foco de atenção, modulação do comportamento e mecanismos de recompensa (Levy \& Swanson, 2001). Desta maneira, uma disfunção neste sistema correlaciona-se com o que é observado clinicamente no TDAH, principalmente em hiperativos, como, por exemplo, problemas de planejamento.

O sistema noradrenérgico também tem sido relacionado a vários aspectos da atenção e na regulação do sono-vigília. Provavelmente, TDAH e transtornos cognitivos ocorrem devido a uma desregulação do metabolismo de noradrenalina no lócus ceruleus, envolvendo receptores_2 adrenérgicos (Arnsten, Steere \& Hunt, 1996). Este sistema está intimamente associado à modulação de funções corticais elevadas, incluindo a atenção, alerta e funções executivas.

As vias serotoninérgicas têm uma apresentação complexa, distribuindo-se difusamente no cérebro e apresentando vários tipos de receptores. Baixos níveis de serotonina foram correlacionados com impulsividade, transtorno oposicional desafiante e comportamento agressivo (Halperin et al., 1994).

Atualmente, alguns estudos encontraram fortes evidências entre o TDAH e componentes hereditários (Milberger, Biederman, Faraone, Chen, \& Jones, 1996; Roman, Rohde, \& Hutz, 2002). Entretanto, até o presente momento, as pesquisas não identificaram um gene principal, mas sim, possíveis genes de efeito pequeno ou moderado (Wohl, Purper-Ouakil, Mouren, Adés, \& Gorwood, 2005). Esses genes de risco ou vulnerabilidade apenas aumentariam as chances de uma criança vir a apresentar os sintomas do TDAH ou de apresentar os sintomas do transtorno durante a vida adulta.

Vários fatores psicossociais parecem estar associados ao desenvolvimento do TDAH muito embora ainda não existam evidências concretas de uma relação causal entre estes agentes e o transtorno (Harada, Yamazaki, \& Saitoh, 2002). Os principais fatores incluem condição social, instabilidade familiar e discórdia marital, estilo parental, qualidade das interações familiares e psicopatologia materna (Biederman et al., 1995; Harvey, 1998; Hinshaw, Zupan, Simmel, Nigg, \& Melnick, 1997; Oosterlaan \& Sergeant, 1996; White, 1999). Recentemente, Vasconcelos et al. (2005) analisaram a contribuição dos fatores psicossociais para a ocorrência do TDAH em uma amostra composta de 403 alunos de 6 a 15 anos. A adversidade psicossocial estatisticamente significativa foi "brigas conjugais no passado". Segundo os autores, crianças expostas a esta variável correram um risco quase 12 vezes maior de ter o diagnóstico do transtorno.

\section{Diagnóstico}

O diagnóstico do TDAH nem sempre é uma tarefa fácil, haja vista que sua fundamentação está pautada em critérios clínico-comportamentais. Assim sendo, o diagnóstico é dependente da história clínica do paciente, informe dos pais e da escola, uso de questionários associados aos critérios apontados por sistemas classificatórios formais. Dentre eles, destaca-se o SNAP-IV (Power \& Ikeda, 1996), Child Behavior Cheklist (CBCL) (Lampert, Polanczyk, Tramontina, Mardini \& Rohde, 2004) e The Adult Symptom Rating Scale (ASRS) (Kessler et al., 2005) . O DSM-IV é o sistema, atualmente, mais utilizado e reconhece três subtipos de TADH, o tipo combinado, o predominantemente desatento e o hiperativo-impulsivo. Não raro, existem subgrupos de pacientes que apresentam manifestações clínicas comuns a outros quadros neurológicos/psiquiátricos, caracterizadas principalmente pelas comorbidades, que tanto podem estar presentes em indivíduos com TDAH, como também podem ocorrer isoladamente e ou acompanhadas da desatenção, impulsividade ou da hiperatividade.

Segundo Rohde, Filho, Benneti, Gallois e Kieling (2004), mais de 70\% dos pacientes com 
TDAH que procuram ambulatórios especializados apresentam alguma comorbidade. Transtornos de conduta, transtorno desafiador opositivo, transtornos de ansiedade, transtornos da fala e da linguagem e problemas de aprendizagem são as mais comumente diagnosticadas (Gadow, Nolan, Sprafkin, \& Schwartz, 2002; Iwanga, Ozawa, Kawasaki, \& Tsuchida, 2006; Mayes, Calhoun, \& Crowell, 2000; Milberger, Biederman, Faraone, \& Guite, 1997).

Dessa maneira, o tratamento do paciente com TDAH é complexo, multidisciplinar e condicionado por uma grande quantidade de fatores. Dentre eles, destaca-se a idade, o sexo, sintomas clínicos predominantes, ambientes familiar e escolar e nível socioeconômico (Rotta, Ohlweiler \& Riesgo, 2005). Cada indivíduo necessitará de um tratamento que possa contemplar a maior quantidade de variáveis envolvidas na aquisição e/ou manutenção dos sintomas relacionados ao transtorno, levando-se sempre em consideração a possibilidade da presença de alguma comorbidade.

\section{Tratamento Farmacológico}

Centenas de estudos conduzidos indicaram que os estimulantes, certos antidepressivos e a clonidina podem ser de grande utilidade para portadores de TDAH (Purper-Ouakil, 2006; Weiss \& Hechtman, 2006). Rohde e Benczkik (1999) afirmam que vários estudos cuidadosos demonstram claramente que cerca de $70 \%$ das crianças e adolescentes com o distúrbio apresentam melhoras significativas dos sintomas de desatenção, de hiperatividade e impulsividade na escola e em casa com o uso correto de remédios.

\section{Estimulantes}

Os estimulantes são as drogas mais utilizadas, sendo considerados medicações de primeira escolha para o transtorno (Barbaresi et al., 2006). Os três estimulantes mais comumente recomendados para o TDAH são as drogas d-anfetamina (Dexedrina ${ }^{\circledR}$ ), metilfenidato (Ritalina $\left({ }^{\circledR}\right.$ ) e pemolina (Cylert $\left.{ }^{\circledR}\right)$ (Biederman et al., 2005). No Brasil, o único estimulante encontrado no mercado é o metilfanidato. Os estimulantes são considerados as medicações mais testadas, sendo que entre os anos de 1983 e 2000, publicaram-se mais de 1400 arti- gos científicos apenas sobre o tratamento com estimulantes (Vasconcelos et al., 2005). A eficácia do metilfenidato já foi comprovada em mais de 1500 estudos clínicos nos últimos 40 anos (Greydanus, 2005). Estas medicações têm se mostrando bastantes eficazes na melhora do comportamento, desempenho acadêmico e ajustamento social para aproximadamente 50 a 95\% das crianças com TDAH (Biederman et al., 2005). Spencer et al. (1996) relataram 155 estudos bem controlados utilizando-se estimulantes com um total de mais de 5700 participantes, dentre eles, crianças, adolescentes e adultos. Estas medicações foram eficazes em aproximadamente em $70 \%$ dos estudos. Segundo os autores, estas drogas não melhoram somente comportamentos relacionados aos transtornos, mas também melhoram a auto-estima e o raciocínio.

\section{Antidepressivos Tricíclicos}

Clinicamente, os antidepressivos tricíclicos (ADT) são indicados nos casos em que não há resposta aos estimulantes e na presença de comorbidade com transtornos de tique ou enurese (Rohde \& Halpern, 2004). Os antidepressivos tricíclicos freqüentemente utilizados no TDAH são desipramina, imipramina e amitriptilina. Existem mais de 25 estudos que apontam a eficácia dos ADT no tratamento do TDAH, muito embora a maioria destas pesquisas restringe-se a crianças em idade escolar (Spencer et al., 2002).

\section{Bupropiona}

A bupropiona é classificada como um antidepressivo atípico inibidor da recaptação de dopamina e noradrenalina. Em uma revisão de literatura sistemática realizada por Segenreich e Mattos (2004) acerca do uso da bupropiona no tratamento do TDAH, observou-se que as limitações metodológicas dos estudos encontrados não permitiram avaliar o grau de eficácia deste fármaco em comparação com medicações de primeira escolha para o tratamento do transtorno. Entretanto, os autores consideram a bupropiona como uma alternativa de tratamento, uma vez que os estudos encontrados indicam existir eficácia deste fármaco. Achados mais recentes também observaram redução dos sintomas do TDAH utili- 
zando-se a bupropiona (Levin et al., 2006; Wilens et al., 2005).

\section{Clonidina}

A clonidina é uma droga utilizada em resposta à intolerância do uso de estimulantes ou na presença de comorbidades, dentre elas o transtorno de conduta. Sua eficácia pode ser comparada à dos antidepressivos tricíclicos (Prince, Wilens, Biederman, Spencer, \& Wozniack, 1996; Wilens, Faraone, Biederman, \& Gunawardene, 2003).

\section{Atomox etina}

Atualmente a atomoxetina é uma nova opção farmacológica para o tratamento do TDAH recentemente aprovada pelo Food and Drug Administration ( FDA) nos Estados Unidos. Trata-se da primeira droga não pertencente à classe dos estimulantes, oficialmente aprovada para tratamento do TDA/H em crianças, adolescentes e adultos. Seu modo de ação se dá pela inibição seletiva da recaptura de noradrenalina e dopamina (Byamaster et al., 2002). Embora não existam ainda muitos estudos acerca da eficácia da atomoxetina, em todas as pesquisas realizadas, este fármaco foi superior ao placebo na redução dos sintomas nucleares do transtorno (desatenção, hiperatividade e impulsividade). (Allen et al., 2005; Biederman, Kratochvil, Spencer, \& Wozniack, 2003; Purper-Ouakil, Fourneret, Wohl, \& Reneric, 2005; Prince, 2006; Shmueli \& Gross-Tsur, 2005; Spencer et al., 2006; Wilens, Kratochvil, Newcorn, \& Gao, 2006).

\section{Tratamento Não-Farmacológico}

Vários estudos têm demonstrado que intervenções não medicamentosas, focadas no contexto psicossocial, escolar e psicoterápico são capazes de auxiliar na redução dos sintomas do TDAH (Safren, Sprich, Chulvick, \& Otto, 2004).

No âmbito psicossocial, inicialmente é importante que se faça o esclarecimento aos pais e familiares acerca do transtorno. Quanto mais informação e conhecimento eles tiverem sobre o TDAH, maiores serão as possibilidades de a família contribuir de maneira efetiva na elaboração de um tratamento que seja mais eficaz e confortável para o portador o distúrbio. Alguns estudos têm encontrado evidências concretas entre a redução dos sintomas do TDAH e programas de treinamento para os pais (Pelham, Wheeler, \& Chronis, 1998; Sonuga-Barke, Daley, Thompson, Laver-Bradbury, \& Weeks, 2001). Rohde e Halpern, (2004) enfatizam que estes programas devem ensiná-los a reforçarem comportamentos adaptativos socialmente e punirem comportamentos disfuncionais de seus filhos. Além do mais, é importante que estes programas contemplem o ensino aos pais de um conjunto de pequenas medidas e atitudes que auxiliam na criação de uma estrutura que facilite o cotidiano do portador do distúrbio (Silva, 2003). Algumas dessas medidas consistem em estabelecer horários para as atividades, organização de cronogramas em relação às obrigações da criança, montar um local de estudo com poucos estímulos visuais e sonoros, entre outros.

O TDAH representa, junto com a dislexia, a principal causa de fracasso escolar de maneira que a dificuldade de aprendizagem está presente em 20\% das crianças com este transtorno (Artigas-Pallarés, 2003, Poeta \& Neto, 2004; SellSalazar, 2003). Geralmente, estes alunos já possuem uma lacuna em seu aprendizado que surge antes do diagnóstico, conseqüentemente, é importante que ele receba o máximo possível de atendimento individualizado. Dada a quantidade de variáveis que podem interferir no processo de aprendizagem, várias possibilidades de intervenção devem ser levadas em consideração no âmbito escolar (American Academy of Pediatrics, 2001). Reiber e McLaughlin (2004) sugerem que uma sala de aula que possa contemplar o aluno com TDAH poderia ser dividida em duas categorias distintas; a primeira refere-se a sua estrutura física, ao passo que a segunda a sua funcionalidade.

Em relação à primeira categoria, estudos têm demonstrado que salas com uma arquitetura fechada (quatro paredes e uma porta) são melhores que salas mais amplas uma vez que sua arquitetura contém menos estímulos visuais e auditivos que impedem a concentração do aluno (Whalen, Henker, Collins, Finck, \& Dotemoto, 1979). Turmas com número reduzido de alunos, até mesmo classes individuais, são opções a mais ao educador para uma otimização do processo de aprendizagem (Rotta et al., 2005). Outro aspecto importante é a posição do aluno na sala de aula. Vários autores afirmam que o aluno com TDAH, quando 
colocado para sentar na primeira carteira, na fila do meio, próximo ao professor, consegue ter sua distração reduzida, o que possibilita um aumento no seu rendimento escolar (Carbone, 2001; Gardi11, DuPaul, \& Kyle, 1996).

A funcionalidade sala de aula também é uma variável importante na otimização do aprendizado do aluno com o transtorno. Yehle e Wambold (1998) afirmam que os aspectos funcionais englobam desde procedimentos usados pelo professor para a transmissão dos conteúdos até a modelagem de comportamentos por meio de reforçamento e punição._Dentro dessa perspectiva, o professor precisa estabelecer rotinas para a realização de tarefas, de forma que estas precisam ser explicadas passo a passo. O planejamento de tempo das atividades não deve ser demasiadamente longo, para que o aluno tenha menor probabilidade de distrair-se. O estabelecimento de regras claras e consistentes também é necessário como forma de reforçar comportamentos adaptativos, punindo aqueles disfuncionais. Estes são procedimentos embasados por uma série de estudos e revisões de literatura (Grandy \& McLaughlin, 1999; Hogan, 1997; Miranda, Presentacion, \& Soriano, 2002; Purvis, Jones, \& Authement, 1992).

No âmbito das intervenções psicoterápicas centradas no portador do distúrbio, a abordagem mais estudada e com maior evidência científica da eficácia na redução dos sintomas nucleares do transtorno é a cognitivo-comportamental (Fechlings, Roberts, Humphries, \& Dawe, 1991; Meichenbaum \& Goodman, 1971; Pelham et al., 2005; Southam-Gerow, Henin, Chu, Marrs, \& Kendall, 1997). Esta abordagem psicoterápica caracterizase pela busca de mudanças nos comportamentos por meio de uma reestruturação cognitiva, substituindo crenças, pensamentos e formas de interpretar as situações, que sejam negativistas e disfuncionais, por outras formas de pensar e agir ( $\mathrm{Sa}-$ fren et al., 2004). Além disso, o indivíduo pode planejar com o terapeuta um conjunto de atividades que incluem desde enfrentamentos graduais de situações, que ele se considera incapaz de realizar, até o estabelecimento de uma agenda que estruture rotinas de atividades que proporcionem prazer e satisfação. Assim, além de ser educado acerca de suas dificuldades, também é instruído a mudar de comportamentos e formas de interpretar e perceber situações que sejam irrealistas e desadaptativas e que estejam contribuindo para manter ou agravar seu problema (Knapp, Johannpeter, Lyszkowski, \& Rohde, 2003).

O foco do processo psicoterápico com o portador de TDAH pode incluir o treino de solução de problemas, treino em habilidades sociais, relaxamento, estabelecimento de agendas de atividades rotineiras de objetivos e reestruturação de formas de pensar e lidar com problemas que podem estar sendo prejudiciais (Silva, 2003).

Os treinos em solução de problemas e em habilidades sociais visam minimizar os comportamentos impulsivos que estejam influenciando negativamente o indivíduo com TDAH. O treino em relaxamento pode ser direcionado para minimizar o impacto da ansiedade e de suas manifestações somáticas, como tensão muscular, tremores e outras sensações desagradáveis. Ele pode incluir reeducação da respiração, da postura e o aprendizado de alguma técnica de relaxamento a ser empregada com regularidade. O estabelecimento de uma agenda de atividades (diária, semanal ou mensal) é muito importante uma vez que sua função é de estruturar as tarefas e atividades de um indivíduo com TDAH.

A reestruturação cognitiva é um dos pilares da terapia. Para um portador de TDAH isso é muito importante, pois a auto-estima costuma ser bastante baixa e ele tende a enxergar a si próprio como incompetente ou inadequado (Silva, 2003). Dessa forma, o terapeuta conduz o indivíduo a reformular alguns conceitos negativos de si mesmo, reduzindo o sofrimento gerado em decorrência das dificuldades enfrentadas em seu cotidiano.

\section{Tratamentos Combinados}

Vários autores têm encontrado uma eficácia maior na redução dos sintomas do TDAH por meio de tratamentos combinados (Safren et al., 2005; Pelham et al, 2005). De acordo com Daley (2004), a consideração de uma grande quantidade de fatores, incluindo comorbidades, características individuais e variáveis ambientais são elementos importantes na elaboração de um plano de tratamento que possa contemplar modalidades de diferentes intervenções.

No final da década de 90, um ensaio clínico realizado pelo The Multimodal Treatment Study of Children With Attention-Deficit/Hyperactivity Disorder Cooperative Group (1999) (The MTA 
Cooperative Group) foi palco de muitas discussões no meio científico. O grupo acompanhou 579 crianças com TDAH por 14 meses, as quais foram divididas em quatro grupos: tratamento apenas medicamentoso, apenas psicoterápico comportamental com as crianças e orientação para pais e professores, abordagem combinada e tratamento comunitário. Os resultados demonstraram que embora em todos os grupos os sintomas do transtorno tivesse sido reduzido, houve uma eficácia superior da medicação nos sintomas nucleares do TDAH quando comparada à abordagem psicoterápica e ao tratamento comunitário. A abordagem combinada (medicação + abordagem psicoterápica comportamental e orientação aos pais e professores) não resultou em eficácia maior nos sintomas centrais do transtorno comparado à abordagem apenas medicamentosa. Entretanto, estes dados devem ser interpretados de maneira cautelosa uma vez que estes resultados foram obtidos com uma amostra de crianças, sendo necessária a replicação em adolescentes e adultos. Além do mais, este estudo teve limitações metodológicas, como a falta de um grupo controle, fato este que impossibilitou comparações mais complexas. Em relação ao tratamento combinado, os autores da pesquisa relataram que algumas crianças não precisaram mais tomar a medicação, sugerindo que isso tenha ocorrido em decorrência da eficácia terapia comportamental para esta amostra.

\section{Considerações Finais}

O TDAH, embora seja um dos transtornos neuropsiquiátricos mais estudados atualmente, não possui, ainda, sua etiologia totalmente esclarecida. Estudos mais recentes, utilizando equipamentos por imagem demonstraram redução no volume e metabolismo de certas áreas cerebrais, dentre elas, regiões situadas no lobo frontal. Entretanto, esta doença parece ter causas multifatoriais, que vão desde aspectos biológicos até ambientais. Seu diagnóstico ainda está fundamentado em entrevistas e questionários, muito embora a possibilidade de realização de exames de neuroimagem possa ser uma opção em um futuro não muito distante. Em relação às diferentes modalidades de tratamento, o mais indicado é sempre a possibilidade de uma equipe multidisciplinar agindo em diversas variáveis relacionadas ao transtorno.

\section{Referências}

Allen, A. J., Kurlan, R. M., Gilbert, D. L., Coffey, B. J., Linder, S. L., Lewis, D. W., et al. (2005). Atomoxetine treatment in children and adolescents with ADHD and comorbid tic disorders. Neurology, 65(12), 1941-1949.

Aman, C. J., Roberts, R. J., \& Pennington, B. F. (1998). A Neuropsychological Examination of the Underlying Deficit in Attention Deficit Hyperactivity Disorder: Frontal Lobe Versus Right Parietal Lobe Theories. Developmental Psychology, 34(5), 956-969.

Amen, D. G., \& Carmichael, B. D. (1997). Highresolution brain SPECT imaging in ADHD. Annals of Clinical Psychiatry, 9(2), 81-86.

American Academy of Pediatrics. (2001). Clinical Practice Guideline: Treatment of School-Aged Child With Attention Deficit/ Hyperactivity Disorder. Pediatrics, 108(4), 1033-1044.

American Psychiatric Association. (2002). Manual diagnóstico e estatístico de transtornos mentais. Porto Alegre: Artmed.

Arnsten, A. F., Steere, J. C., \& Hunt, R. D. (1996). The contribution of alpha (2)-noradrenergic mechanisms to prefrontal cortical cognitive function: Potential significance for attention-deficit hyperactivity disorder. Archieves of General Psychiatry, 53(5), 448-455.

Artigas-Pallarés, J. (2003). Comorbidad en el transtorno por deficit de atención/ hiperactividad. Revista de Neurologia, 36(1), 68-78.

Barbaresi, W. J., Katusic, S. K., Colligan, R. C., Weaver, A. L., Leibson, C. L., \& Jacobsen, S. J. (2006). Long-term stimulant medication treatment of attention-deficit/hyperactivity disorder: results from a population-based study. Journal of Developmental and Behavioral Pediatrics, 27(1), 1-10.

Biederman, J., Kratochvil, C. J., Spencer, T., \& Wozniack, J. (2003). Determining and achieving therapeutic targets in ADHD. The Journal of Clinical Psychiatry, 64, 265-276.

Biederman, J., Mick, E., Surman, C., Doyle, R., Hammerness, P., Harpold, T., et al. (2005). A randomized, placebo-ontrolled trial of OROS 
methylphenidate in adults with attention-deficit/ hyperactivity disorder. Biology Psychiatry, 59(9), 829-835.

Biederman, J., Milberger, S., Faraone, S. V., Guite, J., \& Warburton, R. (1994). Associations between childhood asthma and ADHD: Issues of psychiatric comorbidity and familiaty. Journal of the American Academy of Child \& Adolescent Psychiatry, 33(6), 842-848.

Biederman, J., Milberger, S., Faraone, S. V., Kiely, K., Guite, J., Mick, E., et al. (1995). Family-environment risk factors for attention-deficit hyperactivity disorder: A test of Rutter's indicators of adversity. Archieves of General Psychiatry, 52(6), 464-470.

Byamaster, F. P., Gehlert, D., Nelson, P., Threlkeld, S., Hemrick-Luecke, J., Katner, J., et al. (2002). Atomoxetine increases cortical levels of norepinephrine and dopamine: A proposed mechanism of action in ADHD. European Neuropsychopharmacology, 12, 418-418.

Carbone, E. (2001). Arranging the classroom with an eye (and ear) to students with ADHD. Teaching Exceptional Children, 34(2), 72-81.

Castellanos, F. X., Lee, P. P., Sharp, W., Jeffries, N. O., Greenstein, D. K., Clasen, L. S., et al. (2002). Developmental trajectories of brain volume abnormalities in children and adolescents with attention-deficit/hyperactivity disorder. Journal of American Medical Association, 288(14), 1740-1748.

Daley, E. (2006). Attention deficit hyperactivity disorder: A review of the essential facts. Child: Care, Health and Development, 32(2), 193204.

Daley, K. C. (2004). Update on attention-deficit/ hyperactivity disorder. Current Opinion in Pediatrics, 16(2), 217-226.

Excoffier, E. (2006). What is child attention deficit hyperactivity disorder? Revue du Praticien, 56(4), 371-380.

Fabiano, G. A., \& Pelham, W. E. (2003). Improving the effectiveness of behavioral classroom interventions for attention-deficit/hyperactivity disorder: A case study. Journal of Emotional and Behavioral Disorders, 11(2), 122-131.
Fechlings, D. L., Roberts, W., Humphries, T., \& Dawe, G. (1991). Attetion deficit hyperactivity disorder: Does cognitive behavioral therapy improve home behavior? Journal of Developmental Behavioral Pediatrics, 12(4), 223-228.

Gadow, K. D., Nolan, E. E., Sprafkin, J., \& Schwartz, J. (2002). Tics and psychiatric comorbidity in children and adolescents. Developmental Medicine and Child Neurology, 44(5), 330338.

Gardill, M. C., DuPaul, G. J., \& Kyle, K. E. (1996). Classroom strategies for managing students with attention-deficit/hyperactivity disorder. Interventions in School and Clinic, 32(2), 89-94.

Grandy, S., \& McLaughlin, T. F. (1999). School interventions for students with attention deficit hyperactivity disorder: Some implications for school personnel. International Journal of Special Education, 14(1), 59-70.

Greydanus, D. E. (2005). Pharmacologic treatment of attention-deficit hyperactivity disorder. Indian Journal of Pediatrics, 72(11), 953-960.

Halperin, J. M., Sharma, V., Siever, L. J., Sewartz, S. T., Matier, K., Wornell, G., et al. (1994). Serotoninergic function in agressive and nonagressive boys with attention deficit hyperactivity disorder. American Journal of Psychiatry, 151(2), 243-248.

Harada, Y., Yamazaki, T., \& Saitoh, K. (2002). Psychosocial problems in attention-deficit hyperactivity disorder with oppositional defiant disorder. Psychiatry and Clinical Neurosciences, 56(4), 365-369.

Harvey, P. (1998). Parental employment and conduct problems among children with attentiondeficit/hyperactivity disorder: An examination of child care workload and parenting well-being as mediating variables. Journal of Social \& Clinical Psychology, 17(4), 476-490.

Hinshaw, S. P., Zupan, B. A., Simmel, C., Nigg, J. T., \& Melnick, S. (1997). Peer Status in boys with and without Attention-Deficit Hyperactivity Disorder: Predictions from overt and covert antisocial behavior, social isolation, and authoritative parenting beliefs. Child Development, 68(5), 880-896. 
Hogan, D. (1997). ADHD: A travel guide to succes. Childhood Education, 73, 158-160.

Iwanga, R., Ozawa, H., Kawasaki, C., \& Tsuchida, R. (2006). Characteristics of the sensory-motor, verbal and cognitive abilities of preschool boys with attention deficit/hyperactivity disorder combined type. Psychiatric and Clinical Neurosciences, 60(1), 37-45.

Kessler, R. C., Adler, L., Ames, M., Demler, O., Faraone, S,. Hiripi, E., et al. (2005). The World Health Organization Adult ADHD Self-Report Scale (ASRS): A short screening scale for use in the general population. Psychological Medicine, 35(2), 245-256.

Knapp, P., Johannpeter, J., Lyszkowski, L. C., \& Rohde, L. A. (2003). Terapia cognitivo-comportamental no TDAH: Manual do terapeuta. Porto Alegre: Artes Médicas.

Lampert, T. L., Polanczyk, G., Tramontina, S., Mardini, V., \& Rohde, L. A. (2004). Diagnostic performance of the CBCL-Attention Problem Scale as a screening measure in a sample of Brazilian children with ADHD. Journal of Attention Disorders, 8(2), 63-71.

Levin, F. R., Evans, S. M., Brooks, D. J., Kalbag, A. S., Garawi, F., \& Nunes, E. V. (2006). Treatment of methadone-maintained patients with adult ADHD: Double-blind comparison of methylphenidate, bupropion and placebo. Drug Alcohol Dependence, 81(2), 137-148.

Levy, F., \& Swanson, J. M. (2001). Timing, space and ADHD: The dopamine theory revisited. The Australian and New Zealand Journal of Psychiatry, 35(4), 504-511.

Mataro, M., Garcia-Sanchez, C., Junque, C., Estevez-Gonzalez, A., \& Pujol, J. (1997). Magnetic resonance imaging measurement of the caudate nucleus in adolescents with attention-deficit hyperactivity disorder and its relationship with neuropsychological and behavioral measures. Archieves of Neurology, 54(8), 963-968.

Mayes, S. D., Calhoun, S. L., \& Crowell, E. W. (2000). Learning disabilities and ADHD: Overlapping spectrum disorders. Journal of Learning Disabilities, 33(5), 417-424.
Meichenbaum, D. H., \& Goodman, J. (1971). Training impulsive children to talk to themselves: A means of developing self-control. Journal of Abnormal Psychology, 77, 115-126.

Milberger, S., Biederman, J., Faraone, S. V., \& Guite, J. (1997). Pregnancy, delivery, and infancy complications and attention deficit hyperactivity disorder: Issues of gene-environment interaction. Biological Psychiatry, 41(1), 65-75.

Milberger, S., Biederman, J., Faraone, S. V., Chen, L., \& Jones, J. (1996). New phenotype definition of attention deficit hyperactivity disorder in relatives for genetic analyses. American Journal of Medical Genetics ( Neuropsychiatry Genetics), 67(4), 369-377.

Miranda, A., Presentacion, M. J., \& Soriano, M. (2002). Effectiveness of a school-based multicomponent program for the treatment of children with ADHD. Journal of Learning Disabilities, 35(6), 546-562.

Monteiro, M. A. (2005). Transtorno de déficit de atenção e hiperatividade no adulto (TDAH): perfil sócio-demográfico e estudo de genes candidatos. Dissertação de Mestrado, Universidade de São Paulo, São Paulo.

Oosterlaan, J., \& Sergeant, J. A. (1996). Inhibition in ADHD, aggressive, and anxious children: A biologically based model for child psychopathology. Journal of Abnormal Child Psychology, 24(1), 19-36.

Organização Mundial da Saúde. (1993). Classificação de transtornos mentais e de comportamento da CID-10: Descrições clínicas e diretrizes diagnósticas. Porto Alegre: Artes Médicas.

Pelham, W. E., MacLean, L. B., Gnagy, E. M., Fabiano, G. A., Coles, E. K., Tresco, K. E., et al. (2005). Transdermal methylphenidate, behavioral, and combined treatment for children with ADHD. Experimental and Clinical Psychopharmacology, 3(2), 111-126.

Pelham, W. F., Wheeler, T., \& Chronis, A. (1998). Empirically supported psychosocial treatments for attention deficit hyperactivity disorder. Journal of Clinical Child Psychology, 27(2), 190205. 
Pliszka, S. R. (2005). The Neuropsychopharmacology of Attention-Deficit/ Hiperactivity Disorder. Biological Psychiatry, 57(11), 1385-1390.

Poeta, L. S., \& Neto, F. R. (2004). Estudo epidemiológico dos sintomas do transtorno do déficit de atenção/ hiperatividade e transtornos de comportamento em escolares da rede pública de Florianópolis usando o EDAH. Revista Brasileira de Psiquiatria, 26(3), 150-155.

Power, T. J., \& Ikeda, M. J. (1996). The clinical utility of behavior rating scales: Comments on the diagnostic assessment of ADHD. Journal of School Psychology, 34(4), 379-385.

Prince, J. B. (2006). Pharmacotherapy of attention-deficit hyperactivity disorder in children and adolescents: Update on new stimulant preparations, atomoxetine, and novel treatments. Child AdolescentPsychiatric Clinic of North America, 15(1), 13-50.

Prince, J. B., Wilens, T. E., Biederman, J., Spencer, T. J., \& Wozniack, J. R. (1996). Clonidine for sleep disturbances associated with attentiondeficit hyperactivity disorder: A systematic chart review of 62 cases. Journal of the American Academy of Child \& Adolescent Psychiatry, 35(5), 599-605.

Purper-Ouakil, D. (2006). Treatment of Attention Deficit/Hyperactivity Disorder. Revue du Praticien, 56(4), 381-388.

Purper-Ouakil, D., Fourneret, P., Wohl, M., \& Reneric, J. P. (2005). Atomoxetine: A new treatment for Attention Deficit/Hyperactivity Disorder (ADHD) in children and adolescents. Encephale, 31(3), 337-348.

Purvis, J. R., Jones, C. H., \& Authement, C. (1992). Attention Deficit Hyperactivity Disorder: Strategies for the classroom. Journal of Special Education, 16, 112-118.

Reiber, C., \& McLaughlin, T. F. (2004). Classroom interventions: Methods to improve academic performance and classroom behavior for students with Attention-Deficit/Hyperactivity Disorder. International Journal of Especial Education, 19(1), 1-15.

Rohde, L., \& Benczick, E. (1999). Transtorno de Déficit de Atenção/Hiperatividade: 0 que é? Como ajudar?. Porto Alegre: Artes Médicas.
Rohde, L. A., Filho, E. C., Benetti, L., Gallois, C., \& Kieling, C. (2004). Transtorno de Déficit de Atenção/ Hiperatividade na infância e na adolescência: Considerações clínicas e terapêuticas. Revista de Psiquiatria Clínica, 31(3), 124-131.

Rohde, L. A., \& Halpern, R. (2004). Transtorno de Déficit de Atenção/ Hiperatividade: Atualização. Jornal de Pediatria, 80(2), 61-70.

Roman, T., Rohde, L. A., \& Hutz, M. H. (2002). Genes de suscetibilidade no Transtorno de Déficit de Atenção e Hiperatividade. Revista Brasileira de Psiquiatria, 24(4), 196-2001.

Rotta, N. T., Ohlweiler, L., \& Riesgo, R. S. (2005). Rotinas em Neuropediatria. Porto Alegre: Artmed.

Safren, S. A., Otto, M. W., Sprich, S., Winnet, C. L., Wilens, T. E., \& Biederman, J. (2005). Cognitivebehavioral therapy for ADHD in medication-treated adults with cotinued symptoms. Behaviour Research and Terapy, 43(7), 831-842.

Safren, S. A., Sprich, S., Chulvick, S., \& Otto, M. W. (2004). Psychosocial treatments for adults with Attention-Deficit Hyperactivity Disorder. Psychiatric Clinics of North America, 27(2), 349-360.

Segenreich, D., \& Mattos, P. (2004). Eficácia da bupropiona no tratamento do TDAH: Uma revisão sistemática e análise crítica de evidências. Revista de Psiquiatria Clínica, 31(3), 117-123.

Sell-Salazar, F. (2003). Síndrome de Hiperactividad y Déficit de Atención. Revista de Neurología, 37(4), 353-358.

Shmueli, D., \& Gross-Tsur, V. (2005). Attention Deficit Hyperactivity Disorder: Pharmacological options that are not "Ritalin". Harefuah, 144(8), 572-576.

Silva, A. B. (2003). Mentes Inquietas: Entendendo melhor o mundo das pessoas distraídas, impulsivas e hiperativas. São Paulo: Gente.

Sonuga-Barke, E. J., Daley, D., Thompson, M., Laver-Bradbury, C., \& Weeks, A. (2001). Parentbased therapies for preschool Attention-Deficit/Hyperactivity Disorder: A randomized, controlled trial with a community sample. Journal of the American Academy of Child \& Adolescent Psychiatry, 40(4), 402-408. 
Southam-Gerow, M. A., Henin, A., Chu, B., Marrs, A., \& Kendall, P. C. (1997). Cognitive behavioral therapy with children and adolescents. Child \& Adolescent Psychiatric Clinics of North America, 6(1), 111-136.

Spencer, T. J., Faraone, S. V., Michelson, D., Adler, L. A., Reimherr, F. W., Glatt, S. J., et al. (2006). Atomoxetine and adult Attention-Deficit/Hyperactivity Disorder: The effects of comorbidity. Journal of Clinical Psychiatry, 67(3), 415-420.

Spencer, T., Biederman, J., Coffey, B., Geller, D., Crawford, M., Bearman, S. K., et al. (2002). A double-blind comparison of desipramine and placebo in children and adolescents with chronic tic disorder and comorbid Attention-Deficit/Hyperactivity Disorder. Archives of General Psychiatry, 59(7), 949-656.

Spencer, T., Biederman, J., Wilens, T., Harding, M., O' Donnel, D., \& Griffin, S. (1996). Pharmacotherapy of Attention-Deficit Hyperactivity Disorder across the life cycle. Journal of the American Academy of Child \& Adolescent Psychiatry, 35(40), 409-428.

The MTA Cooperative Group. (1999). A 14- month randomized clinical trial of treatment strategies for Attention- Deficit/ Hyperactivity Disorder. Archieves of General Psychiatry, 56(12), 1073-1086.

Vaidya, C. J., Bunge, S. A., Dudokovic, N. M., Zalecki, C. A., Elliott, G. R., \& Gabrieli, D. E. (2005). Altered neural substrates of cognitive control in childhood ADHD: Evidences from functional magnetic resonance imaging. American Journal of Psychiatry, 162(9), 1605-1613.

Vasconcelos, M. M., Malheiros, A. A., Werner, J., Brito, A. R., Barbosa, J. B., Santos, I. S., et al. (2005). Contribuição dos fatores de risco psicossociais para o Transtorno de Déficit de Atenção/Hiperatividade. Arquivos de Neuro-Psiquiatria, 63(1), 68-74.

Voeller, K. K. (2004). Attention-Deficit Hyperactivity Disorder (ADHD). Journal Child Neurology, 19(10), 798-814.
Weiss, M., \& Hechtman, L. (2006). A randomized double-blind trial of paroxetine and/or dextroamphetamine and problem-focused therapy for Attention-Deficit/Hyperactivity Disorder in adults. The Journal of Clinical Psychiatry, 67(4), 611-619.

Whalen, C. K., Henker, B., Collins, B. E., Finck, D., $\&$ Dotemoto, S. (1979). A social ecology of hyperactive boys: Medication effects in structured classroom enviroments. Journal of Applied Behavioral Analysis, 12, 65-85.

White, J. D. (1999). Personality, temperament and ADHD: A review of the literature. Personality and Individual Differences, 27(4), 589-598.

Wilens, T. E., Faraone, S., Biederman, J., \& Gunawardene, S. (2003). A meta-analysis of clonidine for symptoms of attention: Deficit Hyperactivity Disorder. Pediatrics, 111(1), 179-185.

Wilens, T. E., Haight, B. R., Horrigan, J. P., Hudziak, J. J., Rosenthal, N. E., Connor, D. F., et al. (2005). Bupropion XL in adults with AttentionDeficit/Hyperactivity Disorder: A randomized, placebo-controlled study. Biology Psychiatry, 57(7), 793-801.

Wilens, T. E., Kratochvil, C., Newcorn, J. H., \& Gao, H. (2006). Do children and adolescents with ADHD respond differently to atomoxetine? Journal of the American Academy of Child and Adolescent Psychiatry, 45(2), 149-157.

Wohl, M., Purper-Ouakil, D., Mouren, M., Adés, J., \& Gorwood, P. (2005). Meta-analysis of candidate genes in Attention-Deficit Hyperactivity Disorder. Encephale, 31(4), 437-447.

Yehle, A. K., \& Wambold, C. (1998). An ADHD success story: Strategies for instructors and students. Teaching Exceptional Children, 30(6), 8-13.
Recebido em/received in: 31/10/2006 Aprovado em/approved in: 06/12/2006 